\title{
Beam dynamics simulation for the Compact Linear Collider drive-beam accelerator
}

\author{
Avni Aksoy, ${ }^{1, *}$ Daniel Schulte, ${ }^{2}$ and Ömer Yavaş ${ }^{1}$ \\ ${ }^{1}$ Ankara University, Physics Engineering Department, Ankara, Turkey \\ ${ }^{2}$ CERN, Geneva, Switzerland
}

(Received 9 March 2011; published 26 August 2011)

\begin{abstract}
In the Compact Linear Collider (CLIC) now being studied at CERN, the rf power which accelerates the main beam is provided by decelerating a high current drive beam. The drive-beam linac has to accelerate a 4.2 A electron beam up to $2.4 \mathrm{GeV}$ in almost fully loaded structures. The pulse contains about 70000 bunches, one in every second rf bucket, and has a length of $140 \mu \mathrm{s}$. The beam stability along the beam line is of concern for such a high current and pulse length. We present different options for the lattice of the linac based on FODO, triplet, and doublet cells and compare the transverse instability for each lattice including the effects of beam jitter, alignment, and beam-based correction.
\end{abstract}

DOI: 10.1103/PhysRevSTAB.14.084402

PACS numbers: 29.27.- a, 41.85.-p

\section{INTRODUCTION}

CLIC [1] is based on a two-beam scheme in which the rf power used to accelerate the main beam (at $12 \mathrm{GHz}$ ) is produced by a second beam (the drive beam, DB) running parallel to the main one [2] through so-called power extraction and transfer structure (PETS). This drive beam has a high current but low energy and is decelerated for producing the rf power.

The CLIC drive-beam linac (DBL) will consist of about 750 structures which are low frequency $(1 \mathrm{GHz})$ and will be almost fully loaded transferring more than $95 \%$ of their input power to the beam. The average energy gain per structure will be $\Delta E \approx 3.4 \mathrm{MeV}$ [3]. The initial beam energy is assumed to be $E_{0}=50 \mathrm{MeV}$, the final beam energy $E_{f}=$ $2.4 \mathrm{GeV}$, the bunch charge $q=8.4 \mathrm{nC}$, initial bunch length $\sigma_{z, 0}=3 \mathrm{~mm}$, and the transverse normalized emittances are $\epsilon_{N, x}=\epsilon_{N, y}=50 \mu \mathrm{m}$ [4]. The beam pulse consists of $24 \times 24$ subtrains of about 120 bunches each. The first subtrain fills odd buckets, the immediately following second subtrain fills even buckets; this pattern is then repeated (see Fig. 1). Since the electron source will create uniform charge density along the whole train, first and last bunches of subtrains, in other words the bunches at shifting point from odd to even (even to odd) buckets, will have about half charge of the other bunches that have double spacing.

After DBL, 24 subtrains will be merged into a single subtrain using delay loop (DL), combiner ring one (CR1), and combiner ring two (CR2) [4]. The bunch frequency multiplication will basically be performed by $\mathrm{rf}$ deflectors using phase difference of subtrains. For example, Fig. 2 illustrates subpulse combination scheme by factor 2 on

\footnotetext{
*aaksoy@eng.ankara.edu.tr
}

Published by the American Physical Society under the terms of the Creative Commons Attribution 3.0 License. Further distribution of this work must maintain attribution to the author(s) and the published article's title, journal citation, and DOI.
CLIC DB DL. The phase of bunches filling even buckets will be adjusted to on-crest phase of the deflector cavity operating at $1.5 \mathrm{GHz}$, therefore the bunches filling odd buckets meet negative field inside the cavity. Bunches meeting on-crest phase are sent through a circular path that has a length optimized for length of subpulse. After one turn both bunches of even and odd buckets meet negative field inside deflector and bunches filling even buckets are injected between bunches filling odd buckets. The same routine is repeated on CR1 and CR2 with multiturns and combination factors are 3 and 4, respectively. At the end of CLIC DB complex initial single train with $140 \mu$ s length will be transformed to 24 trains, each has $240 \mathrm{~ns}$ and $100 \mathrm{~A}$ pulse current.

In this study, we discuss major transverse instabilities driven by wakefields in accelerating sections based on different lattice types basically using simulation code PLACET [5]. The linac will be separated into two sections with a bunch compressor which reduces the initial bunch length $\sigma_{z, 0}=3 \mathrm{~mm}$ to the final value $\sigma_{z, f}=1 \mathrm{~mm}$. Currently, the bunch compressor has been simulated with neglecting the impact of imperfections. Also coherent synchrotron radiation has not been included. Additionally, in all calculations we have taken into account only two subtrains 15 bunches each. As it is seen later, the multibunch effects reach steady state condition within this length for a subtrain.

\section{A. Layout of DBL}

In CLIC $1 \%$ luminosity loss requires $\delta \sigma_{\phi} \leq 0.2^{\circ}$ bunch phase and $\delta \sigma_{z} \leq 1 \%$ bunch length jitter in the PETS [6,7].

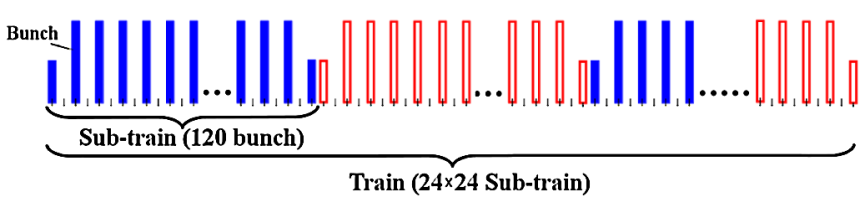

FIG. 1. The charge distributions along the pulse different subtrains are shown in different colors. 


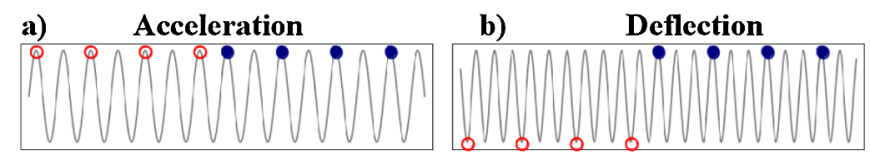

c) Sub-pulse combination

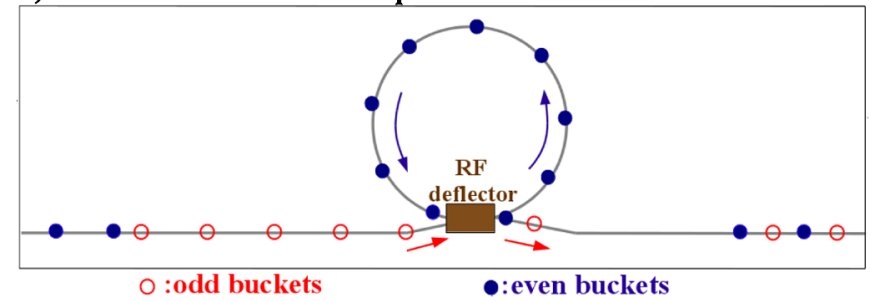

FIG. 2. Illustration of bunch frequency multiplication on CLIC DB DL. (a) Bunch phases with respect to the accelerating field phase on the linac. (b) Bunch phases with respect to the deflecting field phase. (c) Combination scheme.

Therefore bunch energy jitter and bunch phase-length coupling in DBL are of concern. If the full bunch compression is performed in front of PETS, one needs $R_{56} \approx-60 \mathrm{~cm}$ for a chirp of $0.5 \%$ energy spread per $3 \mathrm{~mm}$ bunch length. In that case, for getting acceptable beam phase jitter, one would need $3 \times 10^{-5}$ beam energy jitter. In order to avoid the strong coupling between energy jitter induced in the drivebeam accelerator and beam phase jitter transformed in the bunch compressor, we propose that the bunches are accelerated to $300 \mathrm{MeV}$ in the first stage of the drive-beam linac (DBL1) and compressed from $3 \mathrm{~mm}$ to a length of $1 \mathrm{~mm}$, which is the length required in PETS, and then accelerated to their final energy of $E_{f}=2.4 \mathrm{GeV}$ (see Fig. 3).

Compressing the bunch before the main part of the acceleration, one can afford having a strong bunch energy chirp and small $R_{56}$, thus a weak coupling between beam energy jitter and beam phase jitter can be obtained. In our case we propose a chirp about $2 \%$ energy spread per $3 \mathrm{~mm}$ bunch length and a bunch compressor that has $R_{56}=$ $-10 \mathrm{~cm}$. Assuming additional improvement by factor 10 for the tolerance of the phase using the feed forward system before PETS, the energy error in DBL1 relaxes up to $1 \times 10^{-3}$ [6]. In the second stage of the drive-beam linac (DBL2), the large relative energy spread will be reduced below $0.4 \%$ which is acceptable in the PETS. In order to reduce significant impact of coherent synchrotron radiation, the bunches are uncompressed to $2 \mathrm{~mm}$ before they enter the delay loop and recompressed behind the combiner rings to the final required length of $1 \mathrm{~mm}$. To avoid an energy jitter from DBL2 turning into beam phase jitter, the sum of all $R_{56}$ of all elements after DBL has to be zero. An $R_{56}$ of $1 \mathrm{~mm}$

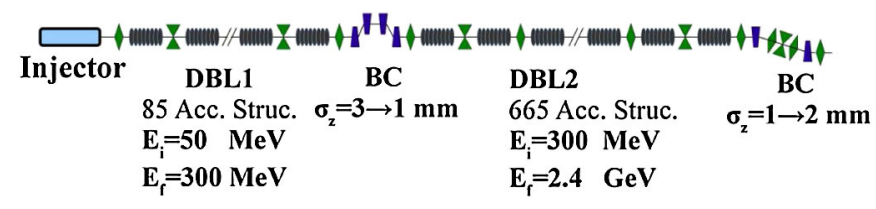

FIG. 3. Basic layout of CLIC drive-beam linac.

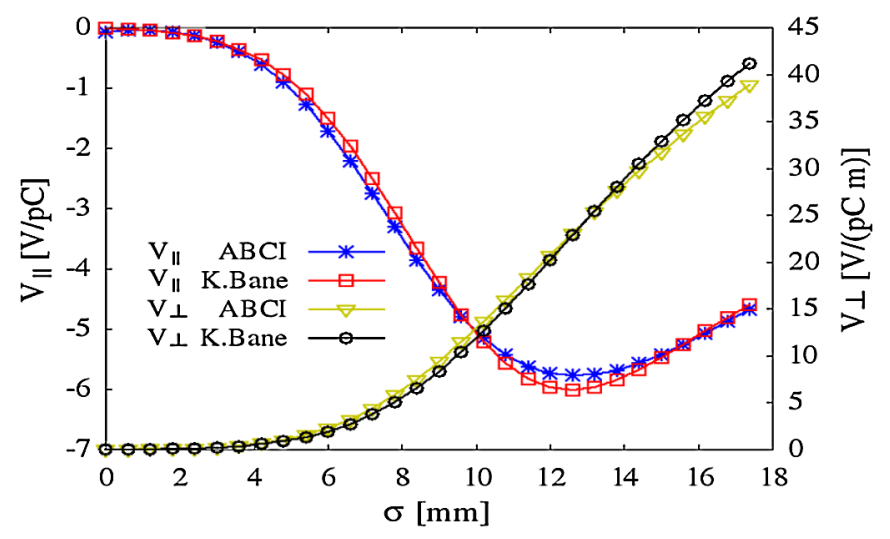

FIG. 4. Wake potentials of a Gaussian bunch with $3 \mathrm{~mm}$ bunch length.

from rest of the beam line and $0.1 \%$ energy error from DBL2 would lead to an acceptable $1 \mu \mathrm{m}$ jitter.

\section{B. Accelerating structure}

The accelerating structure, which will be fed with $15 \mathrm{MW}$ input power, will consist of 19 cells in a length of $2.4 \mathrm{~m}$. It will be the same slotted iris constant aperture structure as in CTF3 [3,8]. Short range wake longitudinal and transverse potentials of the structure have been calculated using $\mathrm{ABCI}$ code [9] for a Gaussian bunch. In order to compute nonGaussian bunch wake especially in DBL2, short range wake functions of the structure have been obtained with numerical fitting of Karl Bane's expressions [10,11] to ABCI results. Figure 4 shows computed wake potentials of a Gaussian bunch using Bane's formulas and ABCI code.

The long-range transverse wakefields used in calculations have been obtained by scaling the lowest four dipole modes of $3 \mathrm{GHz}$ CTF3 structure to $1 \mathrm{GHz}$ [12]. However, the loss and damping factors used in the simulation are 50\% larger than in Ref. [4]. The scaled long-range transverse wakefield modes of some cells of CTF3 structure are given in Table I. Almost perfect compensation of the longrange longitudinal wakefields is predicted [3].

TABLE I. Scaled long-range transverse wakefield modes.

\begin{tabular}{|c|c|c|c|}
\hline $\begin{array}{l}\text { Cells } \\
\#\end{array}$ & $\begin{array}{l}\text { Frequency } \\
f[\mathrm{GHz}]\end{array}$ & $\begin{array}{c}\text { Kick factor } \\
k_{\perp}\left[\mathrm{kV} /\left(\mathrm{pCm}^{2}\right)\right]\end{array}$ & $\begin{array}{c}\text { Quality factor } \\
\qquad Q\end{array}$ \\
\hline \multirow[t]{4}{*}{ First cell } & 1.37 & 6.77 & 8.74 \\
\hline & 1.45 & 9.84 & 8.11 \\
\hline & 1.73 & 2.53 & 71.55 \\
\hline & 1.83 & 0.53 & 3.24 \\
\hline \multirow[t]{4}{*}{ Middle cell } & 1.37 & 6.68 & 6.30 \\
\hline & 1.42 & 12.55 & 3.54 \\
\hline & 1.79 & 1.99 & 59.88 \\
\hline & 1.82 & 0.56 & 3.31 \\
\hline \multirow[t]{4}{*}{ Last Cell } & 1.34 & 14.73 & 5.61 \\
\hline & 1.49 & 4.06 & 20.26 \\
\hline & 1.81 & 1.01 & 5.15 \\
\hline & 1.82 & 2.83 & 3.27 \\
\hline
\end{tabular}




\section{LATTICES}

One of the major problems with accelerating intense bunches in CLIC DBL is the instability driven by wakefields. This instability generated by off-axis beam trajectories can be developed within a single bunch or along a train of bunches and always leads the emittance growth or beam losses. As the beam traverses down along the linac, the head of a single bunch (or first bunch of a train of pointlike bunches) undergoes an unperturbed transverse motion. The tail (or remaining bunches of train), on the other hand, experiences deflection due to the wake excited by the preceding particles (bunches). The amplitude of the deflection in normalized transverse coordinates will be

$$
\Delta x_{N} \propto \int_{0}^{L} \frac{\beta\left(s^{\prime}\right)}{E\left(s^{\prime}\right)} d s^{\prime}, \quad \Delta x_{N}^{\prime} \propto \int_{0}^{L} \frac{\beta\left(s^{\prime}\right)}{E\left(s^{\prime}\right)} d s^{\prime},
$$

where $L$ is the linac length, $\beta(s)$ and $E(s)$ are betatron function and energy along the beam line, respectively [13-15]. The normalization of transverse coordinates at position $s$ on the beam line is given with

$$
\left(\begin{array}{l}
x_{N}(s) \\
x_{N}^{\prime}(s)
\end{array}\right)=\sqrt{\frac{\gamma(s)}{\beta_{x}(s)}}\left(\begin{array}{cc}
1 & 0 \\
\alpha_{x}(s) & \beta_{x}(s)
\end{array}\right)\left(\begin{array}{c}
x(s) \\
x^{\prime}(s)
\end{array}\right),
$$

where $\gamma$ is the relativistic factor, $\beta_{x}$ is the betatron function, and $\alpha_{x}$ is the alpha function of the beam at the position $s$. Equation (2) removes the correlation between $x$ and $x^{\prime}$; in other words, the coordinates have been normalized so that the trajectory of the unperturbed motion, a pure betatron oscillation, is a circle.

Three different lattices were investigated with taking into account their cost. One consists of simple FODO cells, with one structure between each pair of quadrupoles. The other lattice is based on doublets in which two structures are placed in one cell. The last one is the triplet which houses two structures similar to a doublet (see Fig. 5). Equation (1) requires small betatron functions especially when the beam energy is small. Therefore we have optimized lattices for minimum integration and the best phase advance along the beam line. With constant quadrupole spacing and with constant phase advance per cell, the strengths of quadrupoles reach to high values by the end of beam line (e.g. $\sim 0.65 \mathrm{~T}$ pole tip field for $22 \mathrm{~cm}$ quadrupole length).

In the FODO lattice, the length of each quadrupole is $20 \mathrm{~cm}$, the spacing between quadrupoles is $2.9 \mathrm{~m}$, and the phase advance is $\mu_{x, y}=103^{\circ}$ per cell. The doublet lattice has phase advance of $\mu_{x, y}=58^{\circ}$, the doublet spacing is $5.4 \mathrm{~m}$, the distance between two quadrupoles is $40 \mathrm{~cm}$, and the length of each quadrupole is $20 \mathrm{~cm}$. In triplet, the distance between triplets is $5.4 \mathrm{~m}$, the distances between quadrupoles in triples are $40 \mathrm{~cm}$, and one has $\mu_{x}=46^{\circ}$ and $\mu_{x}=49^{\circ}$ phase advances. For the triplet, the lengths of central and outer quadrupoles are chosen 22 and $16 \mathrm{~cm}$, respectively, also the strength of central one is larger than
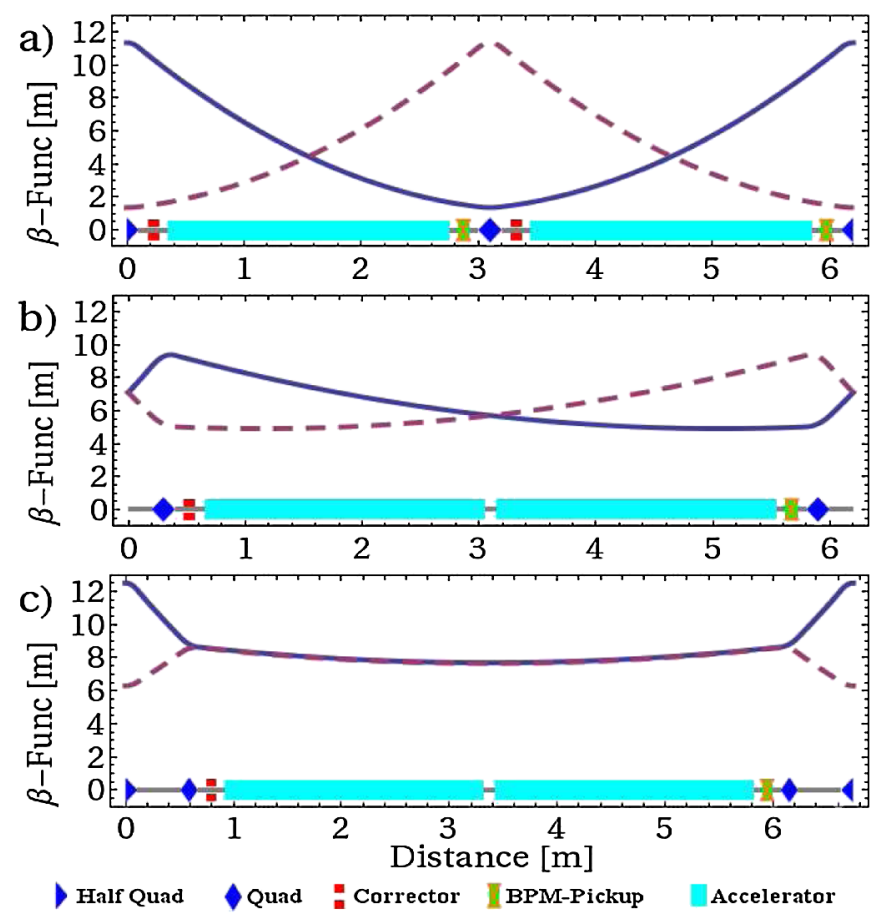

FIG. 5. Sketch of the lattice cells and betatron functions along the cells. (a) FODO. (b) Doublet. (c) Triplet. Strength of quadrupoles is scaled with energy.

the outer ones in order to have equal horizontal and vertical betatron functions inside the structure (see Fig. 5). The lengths of the lattices are comparable but, obviously, the triplet would have more cost due to one more quadrupole for each accelerating structure.

In all following calculations tracking has been started and finished at the middle of the distance between two quadrupoles for FODO and it is the middle of the distance between doublets and triplets for other relevant types of lattices. This choice gives availability to align all quadrupoles in misalignment studies since the alignment is performed respect to the following beam position monitor (BPM) after quadrupole. The nominal Twiss parameters of the beam at the mentioned locations for the considered lattices are

FODO : $\beta_{x}=\beta_{y}=4.44 \mathrm{~m}, \quad \alpha_{x}=-\alpha_{y}=1.66$

doublet: $\beta_{x}=\beta_{y}=5.69 \mathrm{~m}, \quad \alpha_{x}=-\alpha_{y}=0.40$

triplet : $\beta_{x} \approx \beta_{y} \approx 7.64 \mathrm{~m}, \quad \alpha_{x} \approx \alpha_{y} \approx 0.001$.

Since the beam energy is small at the beginning of the DB linac, minimum transverse acceptance will occur at the injection lattice cells just after DB injector. The minimum transverse acceptances are $5.33 \sigma, 6.20 \sigma$, and $5.04 \sigma$ for the FODO, doublet, and triplet lattice, respectively.

\section{TRANSVERSE BEAM JITTER}

Since we cannot estimate the transverse jitter of the incoming beam, only the jitter amplification is calculated. 
The normalized amplification factor Amp for a slice, that has $\Delta x_{0}$ initial offset, is defined as

$$
A m p_{x}=\frac{1}{x_{N}(0)} \sqrt{x_{N}^{2}(L)+x_{N}^{\prime 2}(L)} .
$$

Here, $L$ is length of the beam line, $x_{N}(0), x_{N}(L)$, and $x_{N}^{\prime}(L)$ are initial position, final position, and final angle of the center of the slice in normalized coordinates, respectively. Equivalently, one can define $A m p_{y}$ and the maximum amplification factor $A m p_{\max }$ is the maximum of $A m p_{x}$ and $A m p_{y}$ over all slices. For a slice with nominal energy and without wakefield effects, one has $A m p_{x, y}=1$. However, the beam would also have $\Delta x_{0}^{\prime}$ initial angle or both initial offset and angle together. We would expect to first order that an initial angle transforms in the same way as an initial offset. By considering final offset and angle of a beam, that has an initial offset, we get the full amplification.

In order to look the amplification of bunches in a train, we use the formalism in Ref. [13]. Consider two pointlike bunches with distance $z$ in a train that both are injected with an offset $x_{0}$ into a perfectly aligned machine. The first bunch has unperturbed oscillation through the beam line as $x_{N, 1}(s)=x_{N}(0) \exp \left(-i \int_{0}^{s} \frac{d s^{\prime}}{\beta_{x}\left(s^{\prime}\right)}\right)$. On the other hand, the second bunch experiences deflection due to the wake excited by the driving (first) bunch and its equation of motion is given with

$$
x_{2}^{\prime \prime}+\frac{\gamma^{\prime}(s)}{\gamma(s)} x_{2}^{\prime}+\frac{1}{\beta_{x}^{2}(s)} x_{2}=\frac{N_{1} e^{2}}{E(s)} W(z) x_{1},
$$

where $W$ is the transverse dipole wake at the position of the second bunch created first bunch and $N_{1}$ is number of electrons of the first bunch. For simplification, if we consider $\beta(s), E(s)$, and $W(s)$ do not change over one betatron oscillation, the phase and amplitude can be threaded separately and the excitation induced by the driving bunch can be approximated with a staircase function. For a large number of oscillations and continuously increasing driving function, the solution $x_{2}(s)$ for the second bunch can be simplified as

$$
\begin{aligned}
x_{N, 2}(s) \approx & \left(x_{N}(0)+i \int_{0}^{s} \frac{x_{N, 1} N_{1} e^{2} W\left(z, s^{\prime}\right) \beta_{x}\left(s^{\prime}\right)}{2 E\left(s^{\prime}\right)} d s^{\prime}\right) \\
& \times \exp \left(-i \int_{0}^{s} \frac{d s^{\prime}}{\beta_{x}\left(s^{\prime}\right)}\right) .
\end{aligned}
$$

To expand the case for more than two bunches, we consider $n$ pointlike bunches with distance $z$ in a train that are injected with an offset $x_{j}(0)$ into a perfectly aligned machine. The real part of Eq. (4) corresponds to an oscillation with an offset at some fixed point $s_{0}$ and an imaginary part corresponds to an oscillation with an angle at the same location. We look for only the normalized complex oscillation amplitudes defined with respect to the phase at the beginning of the beam line and ignore the phase factor in Eq. (4). The direct change of the final amplitude $x_{j}(L)$ of bunch $j$ that is induced by the initial offset $x_{k}(0)$ of bunch $k, a_{j-k}$, can be calculated by integrating to the end of linac,

$$
a_{j-k}=i N_{k} e^{2} \int_{0}^{L} \frac{W\left(z_{j}-z_{k}, s^{\prime}\right) \beta\left(s^{\prime}\right)}{2 E\left(s^{\prime}\right)} d s^{\prime} .
$$

Here, $L$ is the length of the linac, $N_{k}$ is the number of electrons of the bunch $k$, and $W\left(z_{j}-z_{k}\right)$ is the transverse wake excited by bunch $k$ and experienced by bunch $j$, which is computed by summing of the modes given in Table $\mathrm{I}$ as given in Ref. [16]. Hence, $x_{N, j}(L)=a_{j-k} x_{N, k}(0)$. However, bunch $j$ can also be effected indirectly by bunch $k$ due to impact of the bunch $k$ to the bunches between $j$ and $k$. We thus define a matrix $A$ which includes all indirect and direct effects such as

$$
A=\exp (a)=\sum_{k=0}^{\infty} \frac{a^{k}}{k !}=\sum_{k=0}^{n-1} \frac{a^{k}}{k !},
$$

where $a$ is a matrix with elements $a_{j k}=a_{j-k}$ for $j>k$ and $a_{j k}=0$ otherwise. Thus, the final offset of all bunches of a train in normalized coordinates $\mathbf{x}_{N}(L)$ will be $\mathbf{x}_{N}(L)=$ $A \mathbf{x}_{N}(0)$.

The impact of an initial offset of a train of bunches with constant charge is shown in Fig. 6. We assumed all bunches have the nominal Twiss parameters and the same initial offset at the entrance of the beam line. In calculation we have used two subtrains of 15 bunches each and for

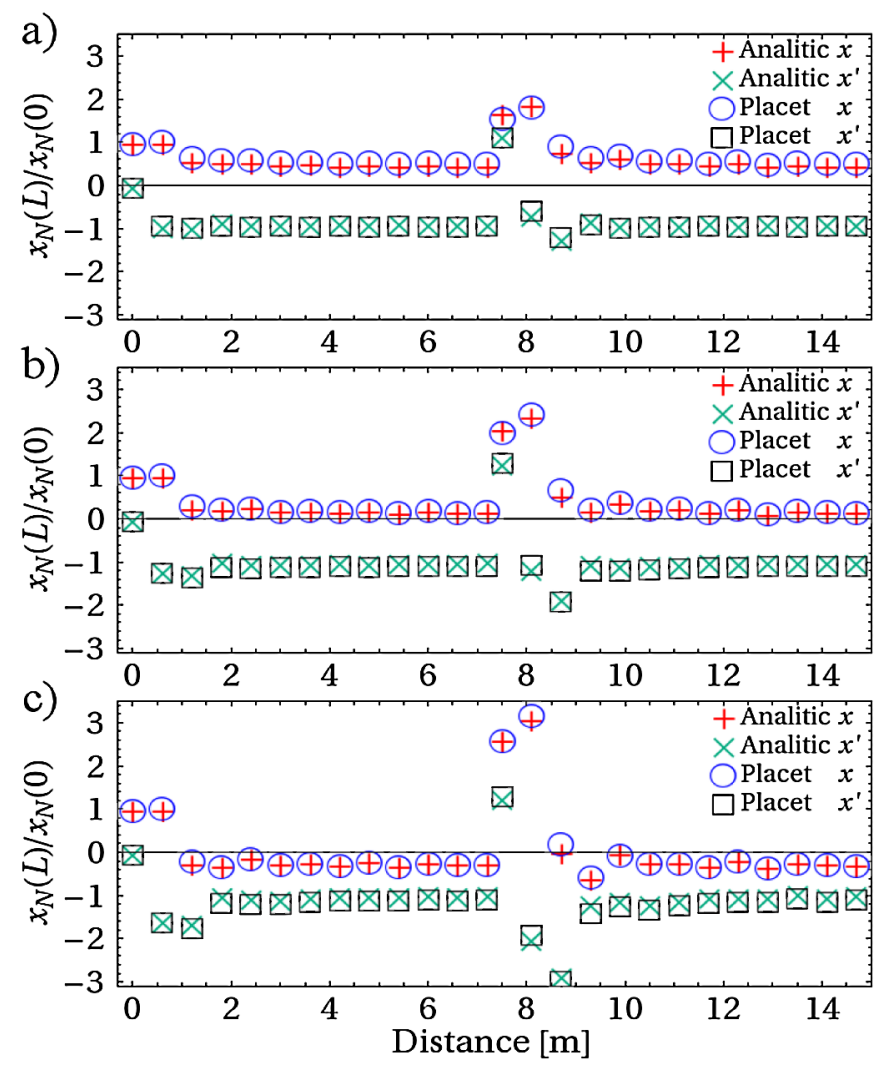

FIG. 6. The normalized amplitudes of the pointlike bunches with constant charge at the end of perfectly aligned DBL for an offset incoming train, (a) FODO, (b) doublet, and (c) triplet. 
checking the worst case we take into account full bunch charge at the subtrain switching point. As it can be seen on the figure, the amplification of bunches of a single subtrain reaches steady state rapidly within this subtrain length and the agreement between the simulation and the simple analytic model is very good. Since the distance between bunches at the switching point from odd buckets to even (or vice versa) is half of the others, the amplification at that point is slightly high due to strong kick caused by closer bunches. the FODO lattice compensates transverse deflections and the worst one occurs on triplet. The maximum amplification factors, $A m p_{\max }$, for the pointlike bunch case for FODO, doublet, and triplet lattices are 2.03, 2.65, and 3.67 , respectively. In the case of the half bunch charge at the subtrain switching point as given on Fig. 1, the amplification factors will decrease to $1.41,1.77$, and 2.34 for FODO, doublet, and triplet lattices, respectively.

For a multiparticle case of bunches, there is additional transverse kick due to short range wakefields. On the other
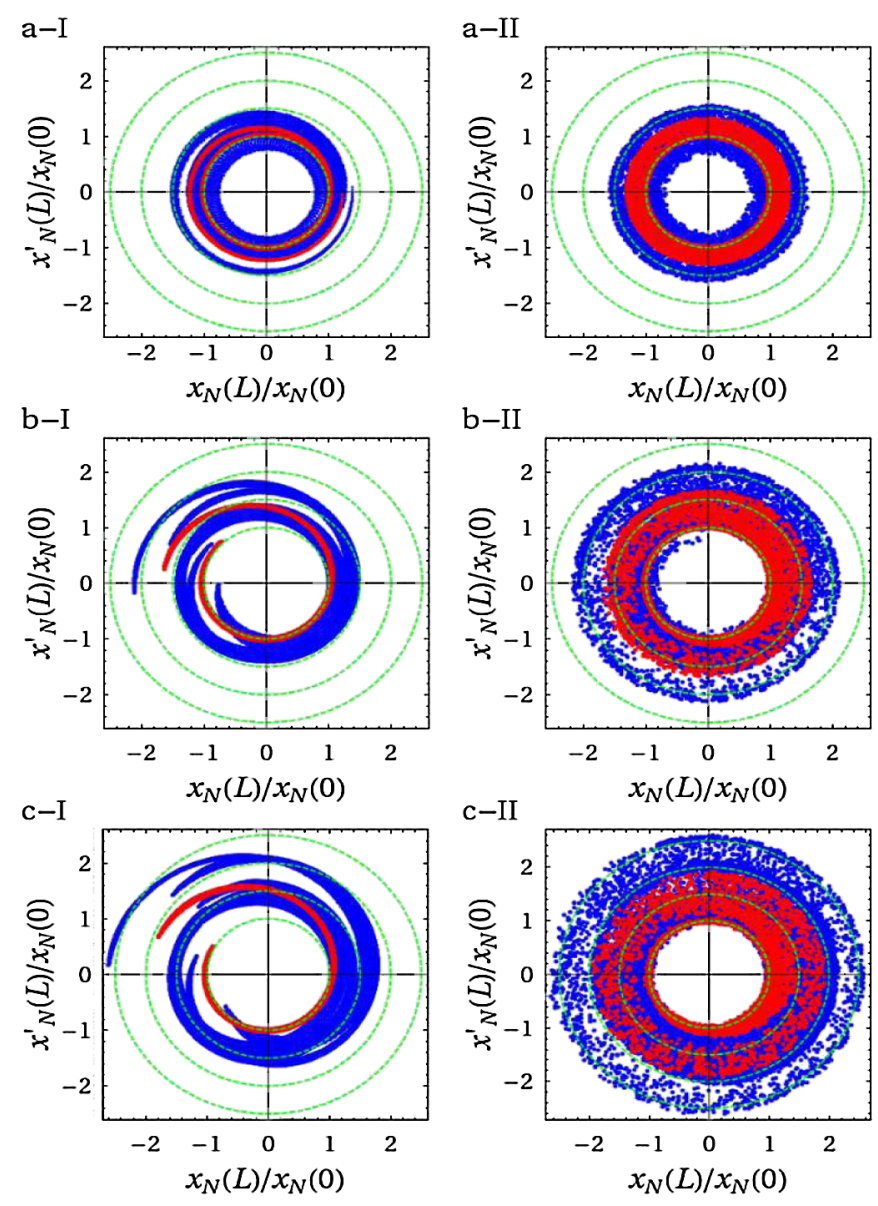

FIG. 7. The normalized final offset of the bunches with constant charge at the end of the DBL1 (I, left-hand side) and DBL2 (II, right-hand side) for an offset incoming train, (a) FODO, (b) doublet, and (c) triplet. Each bunch divided into 51 slices, each slice consists of 101 macroparticles and each dot represents a single macroparticle. The group of red dots shows first bunch and blue dots show trailing bunches. hand, the energy difference of particles within a bunch will force them to advance in phase with respect to the reference one, thus some compensating of the kicks of long-range wakefields occurs [15]. Therefore the amplification factor will not be as high as the pointlike bunches case. Figure 7 shows PLACET results for the final offset of a train at the end of perfectly aligned DBL1 and DBL2. Similar to the pointlike bunches case, all bunches have nominal Twiss parameters at the entrance of the beam line and the train consists of two subtrains 15 bunches each. Switching points from even to odd buckets, the bunches are kicked significantly; the maximum amplification for FODO, doublet, and triplet lattices are $1.55,2.15$, and 2.70, respectively. Without knowledge of the acceptance downstream and the size of the incoming beam jitter, it is not possible to decide whether the amplification is acceptable. For all types of lattices within the linac that have $5 \sigma$ minimum acceptance, even a large incoming jitter of $\Delta x_{0}=\sigma$ does not lead to beam loss.

\section{ALIGNMENT}

All elements on the beam line may be scattered around a straight line. To align the beam line and compute the emittance growth caused by the misalignment, two different routines based on the beam have been taken into account. First, one-to-one correction: Each quadrupole is moved transversely in order to bring the average beam position to zero in the BPM located after quadrupole. Second, wakefield-free steering: Two or more beams with different energy and charge from the nominal one are steered to follow the same trajectory in order to remove dispersion and wakefield effect from the lattice during one-to-one correction is applied to nominal beam [17].

In order to have a better comparison between lattices, we have used one BPM after each quadrupole at an appropriate location (for FODO, after the following structure; for doublet, at the center of the drift between doublets and between structures; for triplet, at the centers of the drifts between triplets and between structures), since one-to-one correction moves quadrupoles for the steering beam to the center of the following BPM.

For DBL1 and DBL2 we have simulated 100 different beam lines, the elements of which are scattered with a normal distribution. In calculation the following assumptions have been considered: (i) all quadrupoles have $\sigma_{x, y}=$ $300 \mu \mathrm{m}$ position errors $\sigma_{x^{\prime}, y^{\prime}}=300 \mu \mathrm{rad}$ angle errors, and $\sigma_{\theta}=1 \mathrm{mrad}$ roll errors; (ii) all BPMs and accelerating structures have only $\sigma_{x, y}=300 \mu \mathrm{m}$ position errors; (iii) the beam line on the bunch compression section is perfectly aligned; (iv) accelerating structures are perfectly straight (no tilting effect); (v) the beam is injected without any offset to DBL1 and DBL2; (vi) the resolution of BPMs are $10 \mu \mathrm{m}$; (vii) each of two test beams used for wakefieldfree steering consists of single bunch and they have $E_{i n, 1}=$ $40 \mathrm{MeV}$ and $E_{i n, 2}=60 \mathrm{MeV}$ initial energies, $Q_{1}=9 \mathrm{nC}$ and $Q_{2}=8 \mathrm{nC}$ charges, $V_{1}=0.93 V_{0}$ and $V_{2}=1.05 V_{0}$ 

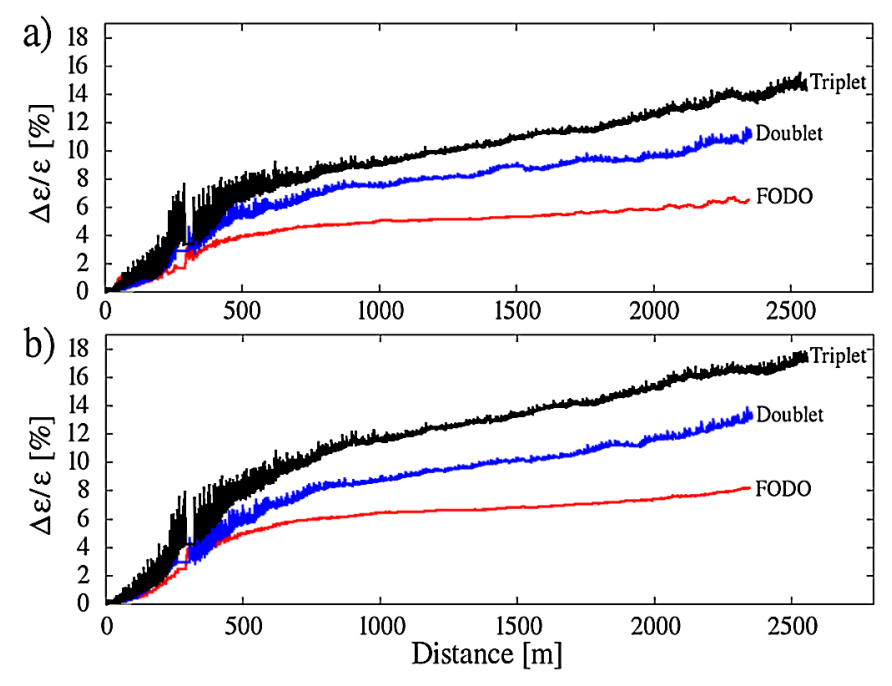

FIG. 8. Emittance growth along the beam line, (a) wakefieldfree steering and (b) one-to-one correction.

accelerating gradients, respectively, where $V_{0}$ is nominal gradient for actual beam.

Figure 8 shows PLACET results for emittance growth along the beam line based on lattices considered. The growth is quite small for all lattice types and for both correction routines. Since the FODO type of lattice has the weakest quadrupoles, the growth on it is smallest and is about $5 \%$. The emittance growth would be higher due to the fact that the bunch compression section will also be misaligned and the beam will have offset at the entrance of DBL2. However, the static imperfection errors given above could be improved somewhat if necessary.

\section{ENERGY AND GRADIENT ERRORS}

Although CLIC DB has very tight tolerances concerning error of incoming beam energy and structure gradients [6], during commissioning large energy and gradient errors may occur. Any error of incoming beam energy or variation of the gradient will lead quadrupole strengths not to be adapted to the beam energy. These situations can cause beam amplification to grow, eventually, beam losses especially in DBL1 where the beam energy is low.

In order to check the amplification, we have simulated the train of two subtrain 15 bunches each on a perfectly aligned beam line of the lattice types considered. We assume the beam has the nominal Twiss parameters and offset of $\Delta x_{0}=\Delta y_{0}=\sigma$ at the entrance of the beam line. Figure 9(a) shows the amplification as a function of the deviation from the nominal initial beam energy (a) and the nominal accelerating gradient (b). As it was discussed in Sec. III minimum amplification is obtained with the FODO lattice for a nominal case. The FODO lattice is more sensitive to initial beam energy variations than the others especially while the beam energy is much lower than the nominal value. The gradient variation more or less does not change the amplification for all lattices and the doublet
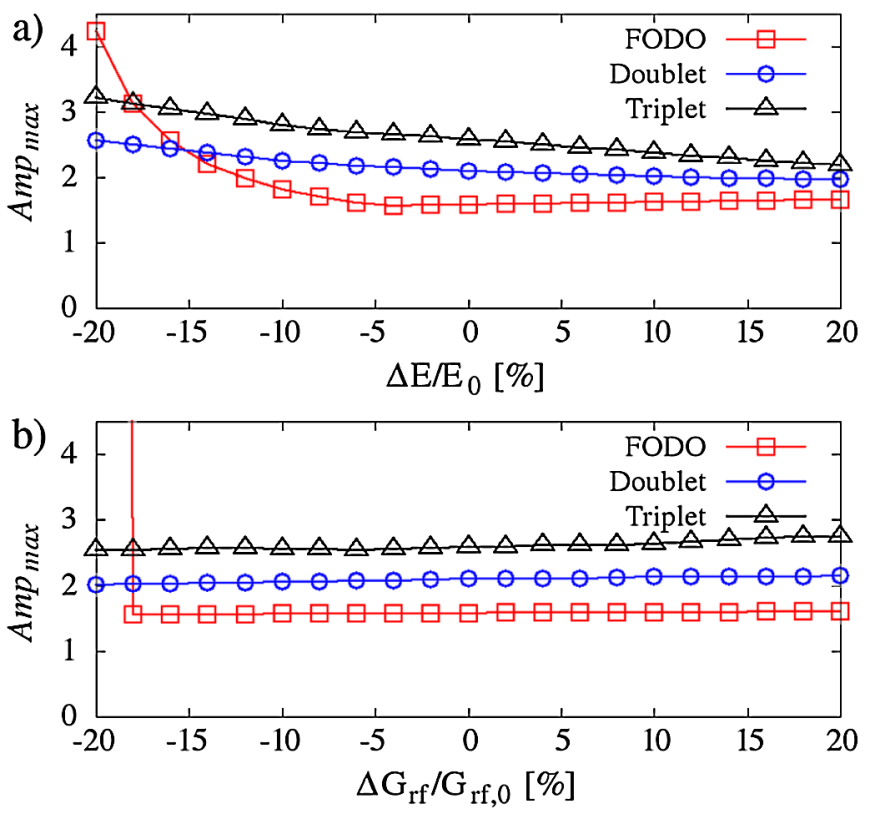

FIG. 9. Amplification of the beam in DBL1, (a) for different initial beam energy and (b) for different accelerating gradient.

lattice seems more stable for both energy and gradient variations. Gradient errors below $-15 \%$ cause amplification to increase rapidly for the FODO type of lattice. This result can be explained as follows: Towards the end of DBL1 the beam, that is accelerated to lower energy than the nominal by low gradient structures, will be overfocused by quadrupoles which are adapted to nominal energy. Thus, the betatron functions will grow rapidly as a result of large phase advance. This situation is not the same for the doublet and the triplet lattice because of small phase advance per cell. Figure 10 shows the amplification of the beam in DBL1 as a function of accelerating gradient error for the FODO type of lattice with different quadrupole settings. As can be seen, smaller phase advance allows larger gradient error.

Another crucial subject would be the acceptance of the lattice in DBL1. The acceptance in normalized coordinates is defined as the beam any particle of which has initial positions $x_{0}, x_{0}^{\prime}, y_{0}$, and $y_{0}^{\prime}$, which fulfills

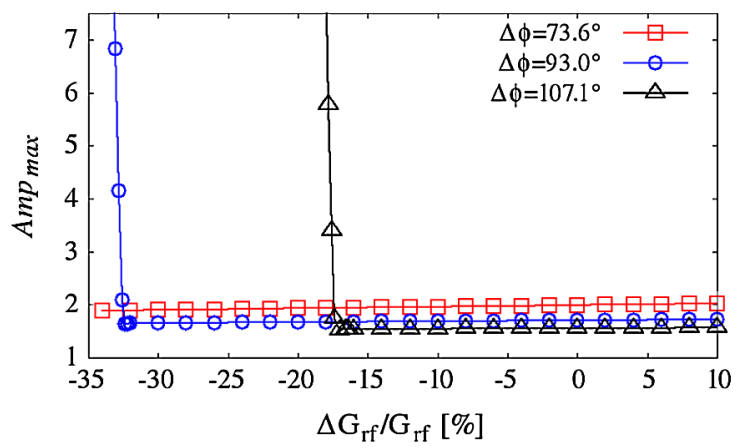

FIG. 10. Amplification of the beam in DBL1 for the FODO type of lattice with different phase advance per one cell. 


$$
A r_{N} \geq \sqrt{x_{N}^{2}+x^{\prime 2}{ }_{N}+y_{N}^{2}+y^{\prime 2}}
$$

will pass through the accelerator. In simulations, we have considered two cases: perfectly aligned beam line and beam line the elements of which are scattered with a normal distribution of $\sigma=300 \mu \mathrm{m}$. For the misaligned case we have simulated 20 different machines and applied one-to-one correction. Additionally, for both cases we assumed the beam which has nominal Twiss parameters has offset $\Delta x_{0}=\Delta y_{0}=500 \mu \mathrm{m}$ at the entrance of the beam line and computed the unnormalized acceptance as

$$
A r_{i}=\min \left(\frac{r_{x, i}-\left|x_{j}\right|}{\sigma_{x}}, \frac{r_{y, i}-\left|y_{j}\right|}{\sigma_{y}}\right),
$$

where $r_{x, i}$ and $r_{y, i}$ are half horizontal and vertical aperture of the element $i, x_{j}$ and $y_{j}$ are the horizontal and vertical position of any macroparticle within the beam, respectively. The acceptance over all beam lines, therefore, will be $A r=$ $\min \left(A r_{i}\right)$. Since the beam has larger size inside the quadrupoles than inside of the other elements, the computation has been performed before and after each quadrupole with setting $40 \mathrm{~mm}$ aperture at both ends of each. We have neglected initial beam angle, expecting that it will transform the same as offset under first order approximation.

Figure 11 shows the acceptance of the lattices as a function of initial beam energy error (a) and accelerating
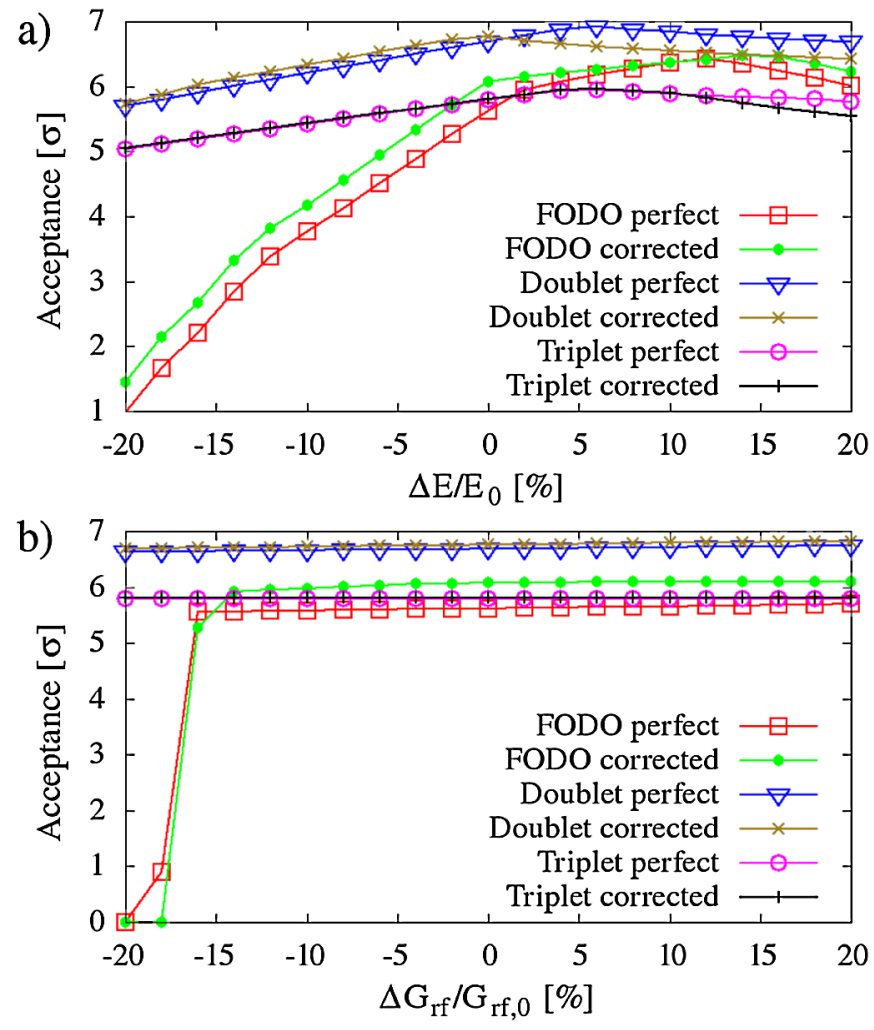

FIG. 11. Acceptance of the lattices, (a) for different initial beam energy and (b) for different accelerating gradient. gradient error (b). The acceptance is highest for the doublet and it is less sensitive to the energy errors in the doublet and the triplet. For FODO the acceptance is reasonable around nominal beam energy, but it is very sensitive to the energy errors due to the same reason explained in amplification calculation. For all lattices the size of the acceptance for perfect and misaligned machines is close to each other. All lattices are less sensitive to the gradient error and the acceptance is highest for the doublet lattice similar to the energy error case. For FODO lattice beam loss starts when the accelerating gradient is below $-15 \%$ of the nominal gradient.

\section{CONCLUSION}

The lattice has to prevent a large amplification of any transverse jitter of the incoming beam. It should also have a large energy acceptance and allow easy correction of static errors of the beam line. Three types of lattice have been studied for finding a compromise between lattices for the CLIC DBL.

The calculations show that, if one uses the FODO type of lattice, the effects of transverse wakefields will be significantly smaller than using doublet or triplet types. For both alignment routines, the triplet type of lattice gives the largest emittance growth. Although FODO and doublet types of lattices have the same number of quadrupoles, FODO gives the smallest emittance growth. On the other hand, the smallest sensitivity to energy errors can be obtained with the doublet type of lattice while the FODO type of lattice yields largest. Nevertheless, too large errors should not be important since the energy of the beam has to be controlled very accurately because of the tight tolerance of energy error in bunch compressor. As it has been discussed in Sec. I A, acceptable energy error is $1 \times 10^{-2}$ for a bunch compressor that has $R_{56}=-10 \mathrm{~cm}$.

The FODO lattice has as many magnets as the doublet solution but fewer than the triplet design. It performs best in terms of jitter amplification and emittance growth in the presence of static imperfections. The energy bandwidth is smaller than it is in the other designs but we consider the first two points more important. In particular, the jitter amplification is very important since this can lead to losses further downstream. This will be a change compared to the CTF3 design, which is based on triplets [12].

The FODO lattice will bring out other advantages such as easy operation. One can also consider using four structures in one FODO cell after $1.5 \mathrm{GeV}$ since the integral given with Eq. (1) will not change dramatically after that energy. For example, if one uses 30\% weaker and $40 \%$ shorter quadrupoles after $1.5 \mathrm{GeV}$, the integral increases from $11.28 \mathrm{~m}^{2} / \mathrm{MeV}$ to $12.439 \mathrm{~m}^{2} / \mathrm{MeV}$ and beam line shortens from 2.35 to $2.21 \mathrm{~km}$. In that case the maximum amplification factor of a train of pointlike bunches will be 2.22 which is still better than the doublet case mentioned above. 
One can also improve the triplet lattice with using single accelerating structure in one triplet cell as it is in the CTF3 design. For instance, with the same quadrupole setting but single structure per triplet cell, the integration given with Eq. (1) reduces from $18.96 \mathrm{~m}^{2} / \mathrm{MeV}$ to $14.25 \mathrm{~m}^{2} / \mathrm{MeV}$. In that case the maximum amplification factor of a train of pointlike bunches can be calculated as 2.58 for the constant bunch charge condition considered in Sec. III. On the other hand, using a single structure per cell would increase the length of beam line from 2.55 to $3.25 \mathrm{~km}$.

[1] R.W. Assmann et al., CERN Technical Report No. CERN-2000-008, 2000.

[2] H. Braun et al., in Proceedings of the 6th European Particle Accelerator Conference, Stockholm, 1998 (IOP, London, 1998), p. 248.

[3] D. Schulte and R. Wegner (personal communication).

[4] H. Braun et al., CERN Technical Reports No. CERNOPEN-2008-021 and No. CLIC-Note-764.

[5] D. Schulte, in Proceedings of the European Particle Accelerator Conference, Vienna, 2000 (EPS, Geneva, 2000), p. 1403; Report No. CERN-PS-2000-028-AE; Report No. CLIC-Note-437, https://savannah.cern.ch/projects/placet/.
[6] D. Schulte et al., CERN Technical Report No. CERNATS-2010-226.

[7] E. Adli, Ph.D. thesis, University of Oslo, Oslo, Norway, 2009.

[8] E. Jensen, I. V. Syratchev, and W. Wuensch, in Proceedings of the 19th IEEE Particle Accelerator Conference, Chicago, 2001 (IEEE, New York, 2001), Vol. 2, p. 855.

[9] Y.H. Chin, KEK Technical Report No. 2005-6.

[10] K. Bane, SLAC Report No. SLAC-PUB-9663.

[11] R. Zennaro, in Proceedings of the 11th European Particle Accelerator Conference, Genoa, 2008 (EPS-AG, Genoa, Italy, 2008), p. 646.

[12] G. Geschonke and A. Ghigo, CERN Technical Reports No. CERN-PS-2002-008-RF, No. CTF-3-NOTE-2002047, and No. LNF-2002-008-IR.

[13] D. Schulte, in Proceedings of the 23rd Particle Accelerator Conference, Vancouver, Canada, 2009 (IEEE, Piscataway, NJ, 2009), p. 4664, FR5RFP055.

[14] K. A. Thompson and R. D. Ruth, Phys. Rev. D 41, 964 (1990).

[15] A. Mosnier, in CERN Accelerator School: 5th Advanced Accelerator Physics Course, edited by S. Turner (CERN, Geneva, 1995), p. 459.

[16] K. L. F. Bane, AIP Conf. Proc. 153, 971 (1987).

[17] T. Raubenheimer, Nucl. Instrum. Methods Phys. Res., Sect. A 306, 61 (1991). 